\title{
Binucleating Hydrazonic Ligands and Their $\mu$-Hydroxodicopper(II) Complexes as Promising Structural Motifs for Enhanced Antitumor Activity
}

\author{
Jesica Paola Rada ${ }^{1}$, Beatriz S. M. Bastos ${ }^{1}$, Luciano Anselmino ${ }^{2}$, Chris H. J. Franco ${ }^{3}$, \\ Mauricio Lanznaster ${ }^{4}$, Renata Diniz ${ }^{5}$, Claudio O. Fernández ${ }^{2}$, \\ Mauricio Menacho-Márquez ${ }^{2}$, Ana Maria Percebom ${ }^{1}$, and Nicolás A. Rey ${ }^{1, *}$ \\ ${ }^{1}$ Pontifical Catholic University of Rio de Janeiro, Rio de Janeiro, Brazil. \\ ${ }^{2}$ Max Planck Laboratory for Structural Biology, Chemistry and Molecular Biophysics of Rosario (MPLbioR, \\ UNR-MPIbpC) and Instituto de Investigaciones para el Descubrimiento de Fármacos de Rosario (IIDEFAR, \\ UNR-CONICET), Universidad Nacional de Rosario, Rosario, Argentina. \\ ${ }^{3}$ Federal University of Juiz de Fora, Juiz de Fora, Brazil. \\ ${ }^{4}$ Fluminense Federal University, Niterói, Brazil. \\ ${ }^{5}$ Federal University of Minas Gerais, Belo Horizonte, Brazil. \\ *nicoarey@puc-rio.br
}

\section{Supporting Information}




\section{Crystals of ligands and metal complexes.}

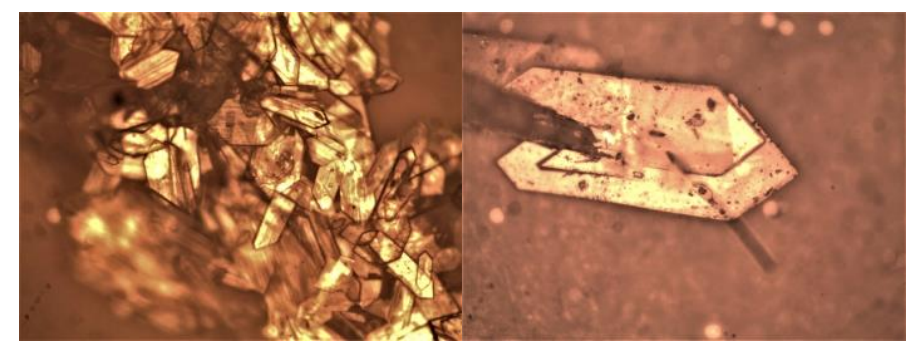

Figure S1. Optical photographs of the $\mathbf{H}_{3} \mathbf{L} \mathbf{1}$ (left) and $\mathbf{H}_{3} \mathbf{L} 2$ (right) crystals.

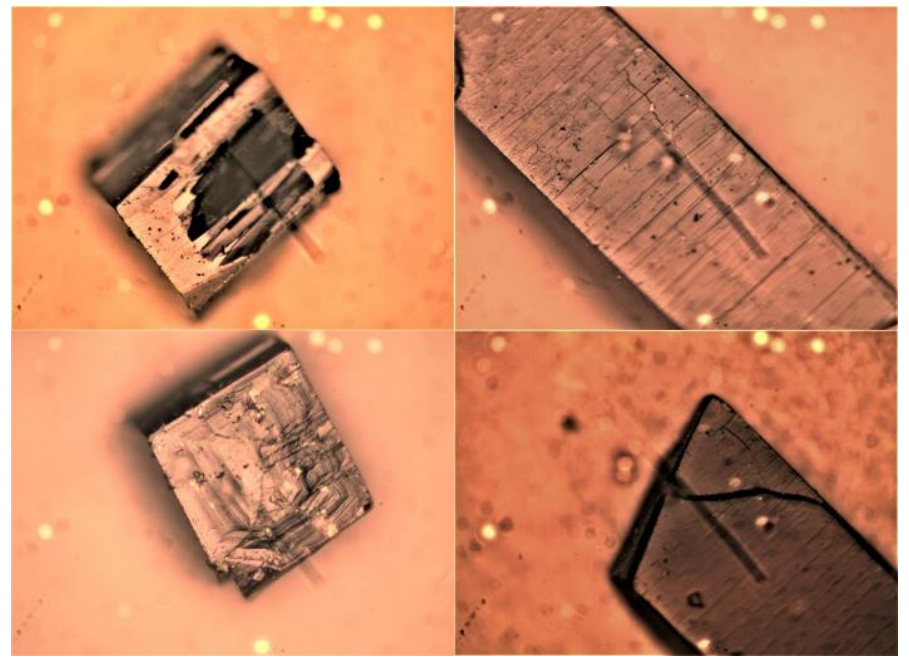

Figure S2. Optical photographs of complex 1 (left) and complex 2 (right) crystals. 
NMR characterization of the ligands.

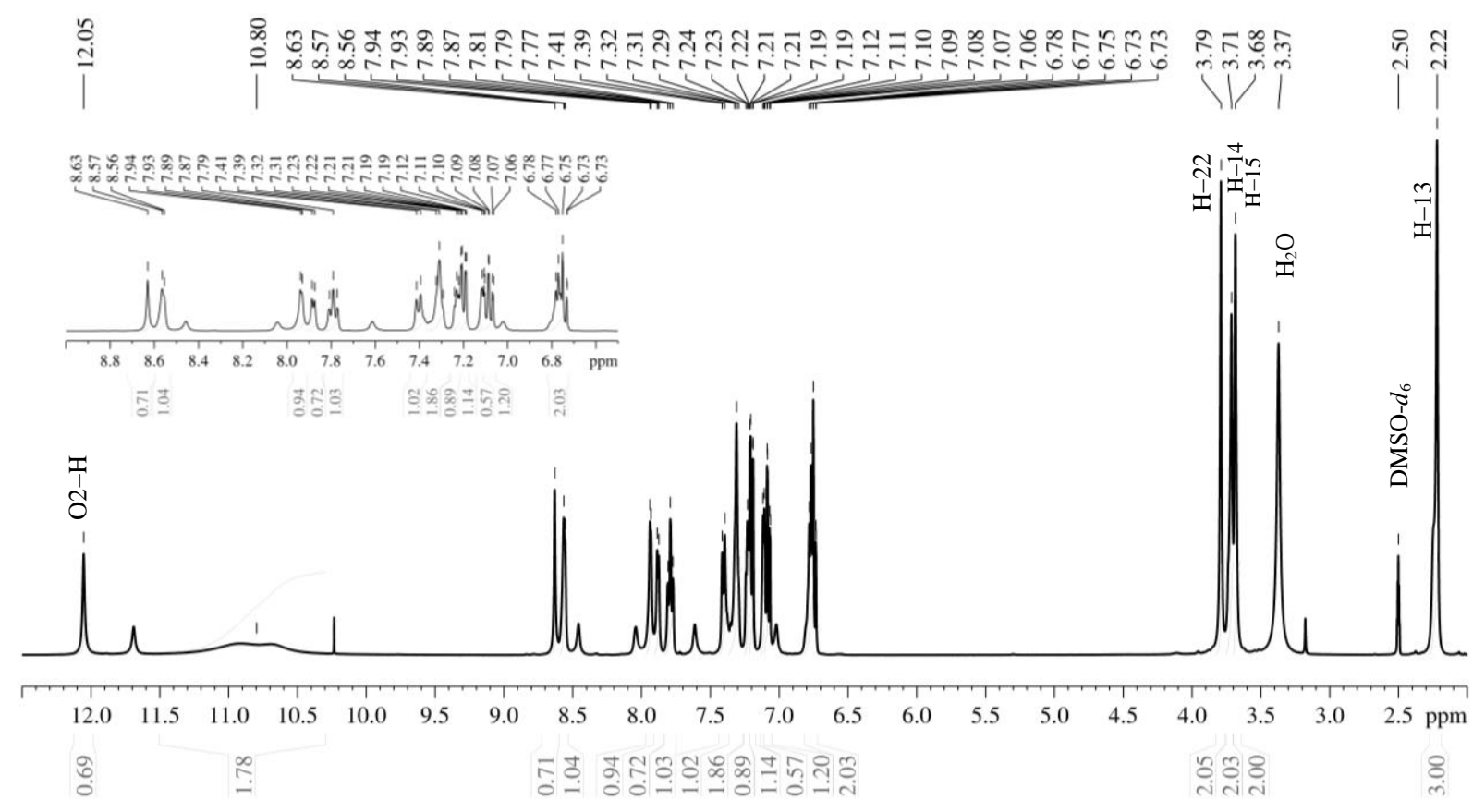

Figure S3. ${ }^{1} \mathrm{H}$ NMR spectra (400 MHz) of $\mathbf{H}_{3} \mathbf{L 1}$ in DMSO- $d_{6}$ at room temperature.

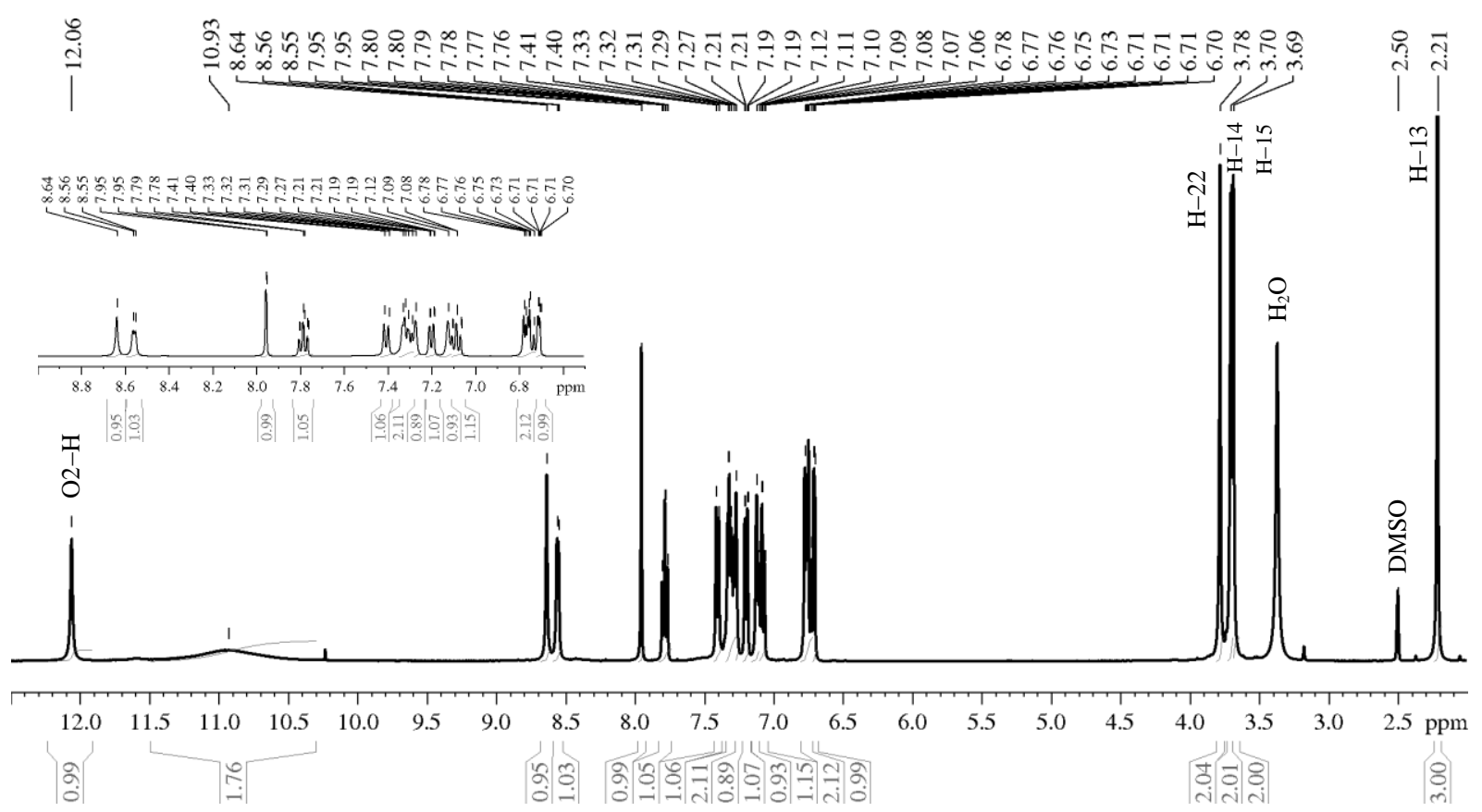

Figure S4. ${ }^{1} \mathrm{H}$ NMR spectra (400 MHz) of $\mathbf{H}_{3} \mathbf{L} \mathbf{2}$ in DMSO- $d_{6}$ at room temperature. 


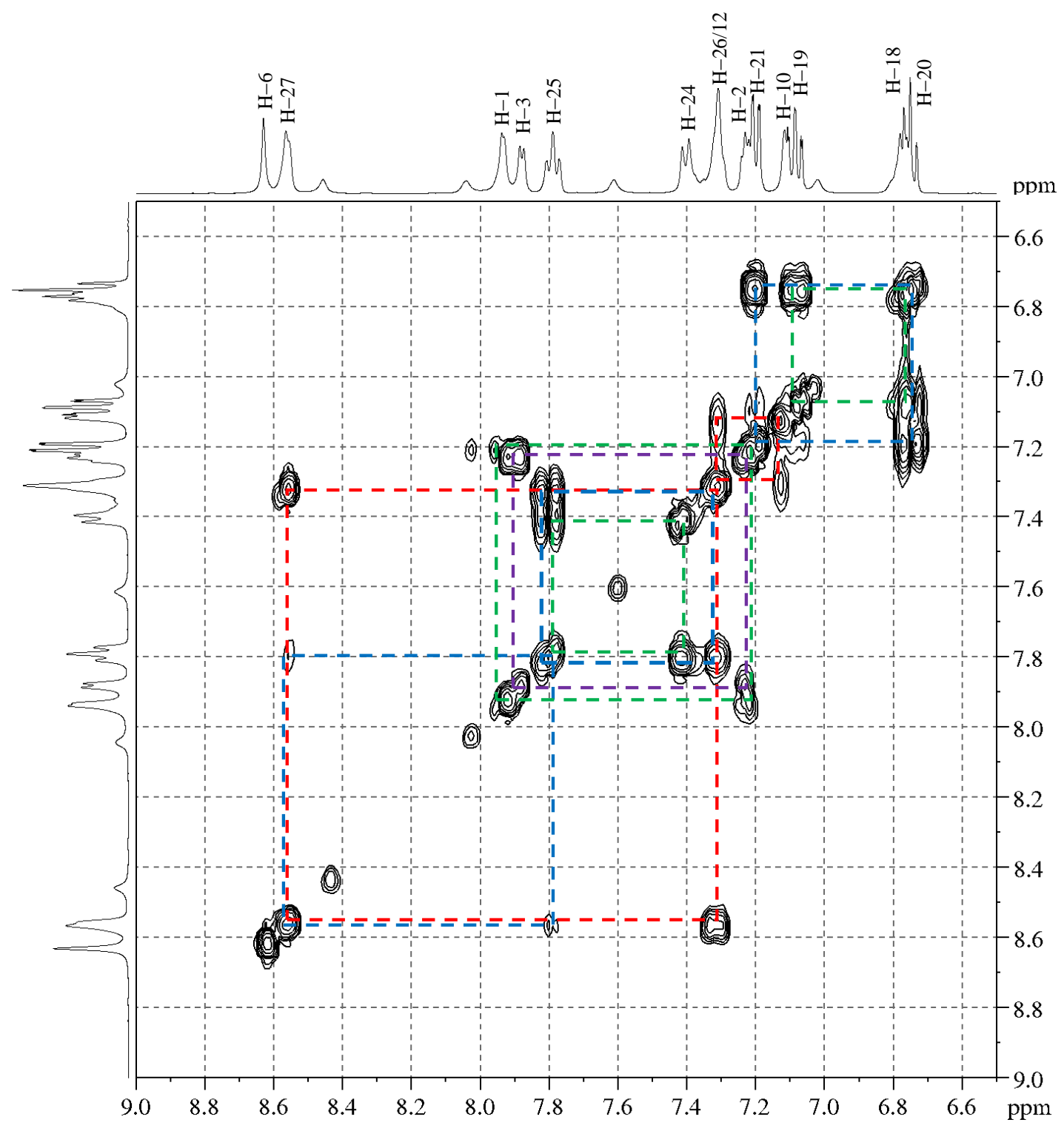

Figure S5. Correlation spectroscopy $\left({ }^{1} \mathrm{H}-{ }^{1} \mathrm{H}\right.$ COSY) NMR contour map (400 MHz) of $\mathrm{H}_{3} \mathrm{L1}$ in DMSO- $d_{6}$ at room temperature. 


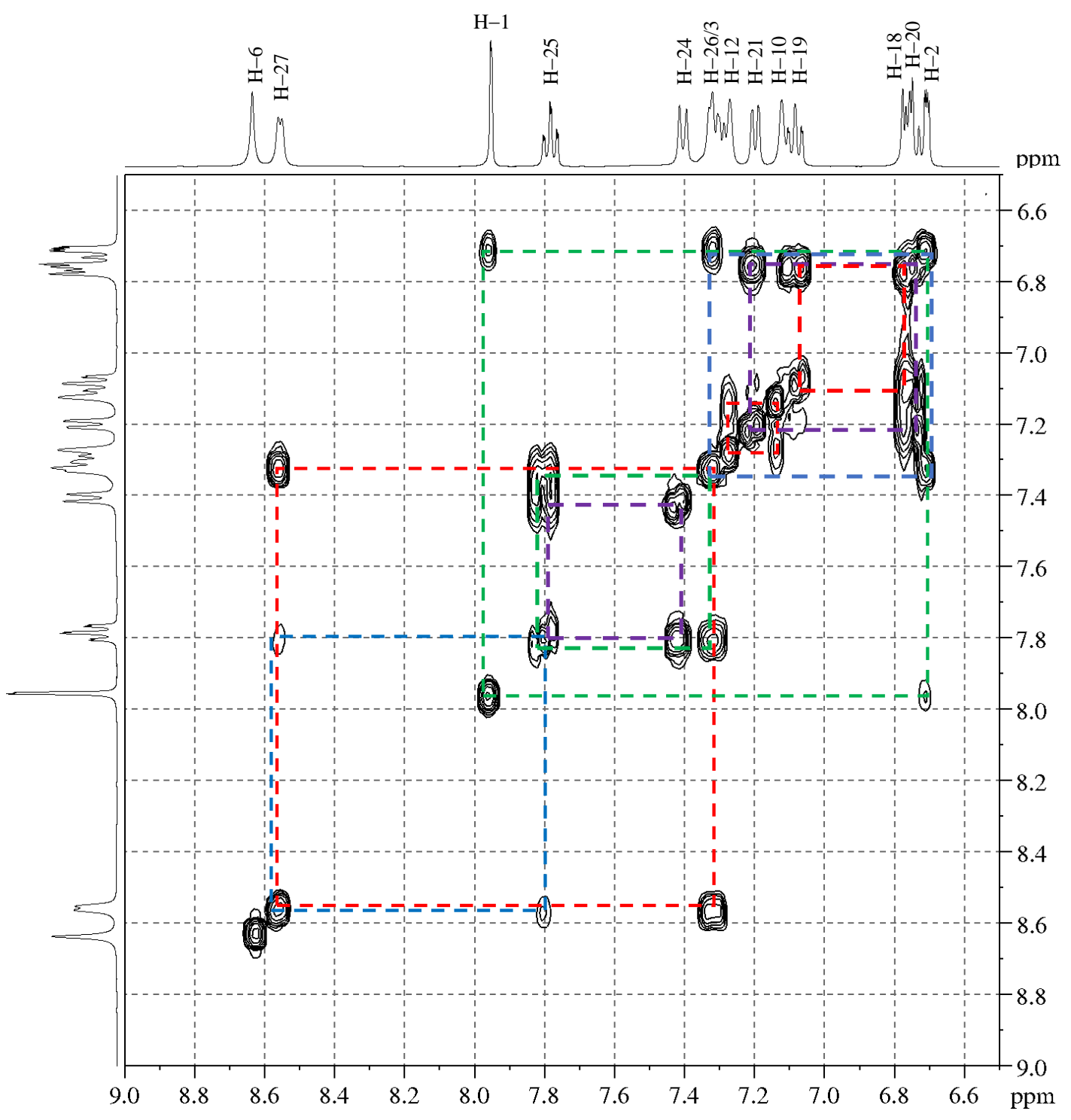

Figure S6. Correlation spectroscopy $\left({ }^{1} \mathrm{H}-{ }^{1} \mathrm{H}\right.$ COSY) NMR contour map (400 MHz) of $\mathrm{H}_{3} \mathbf{L} 2$ in DMSO- $d_{6}$ at room temperature. 


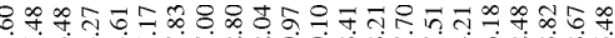

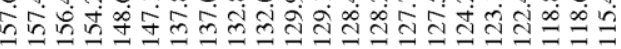

1112

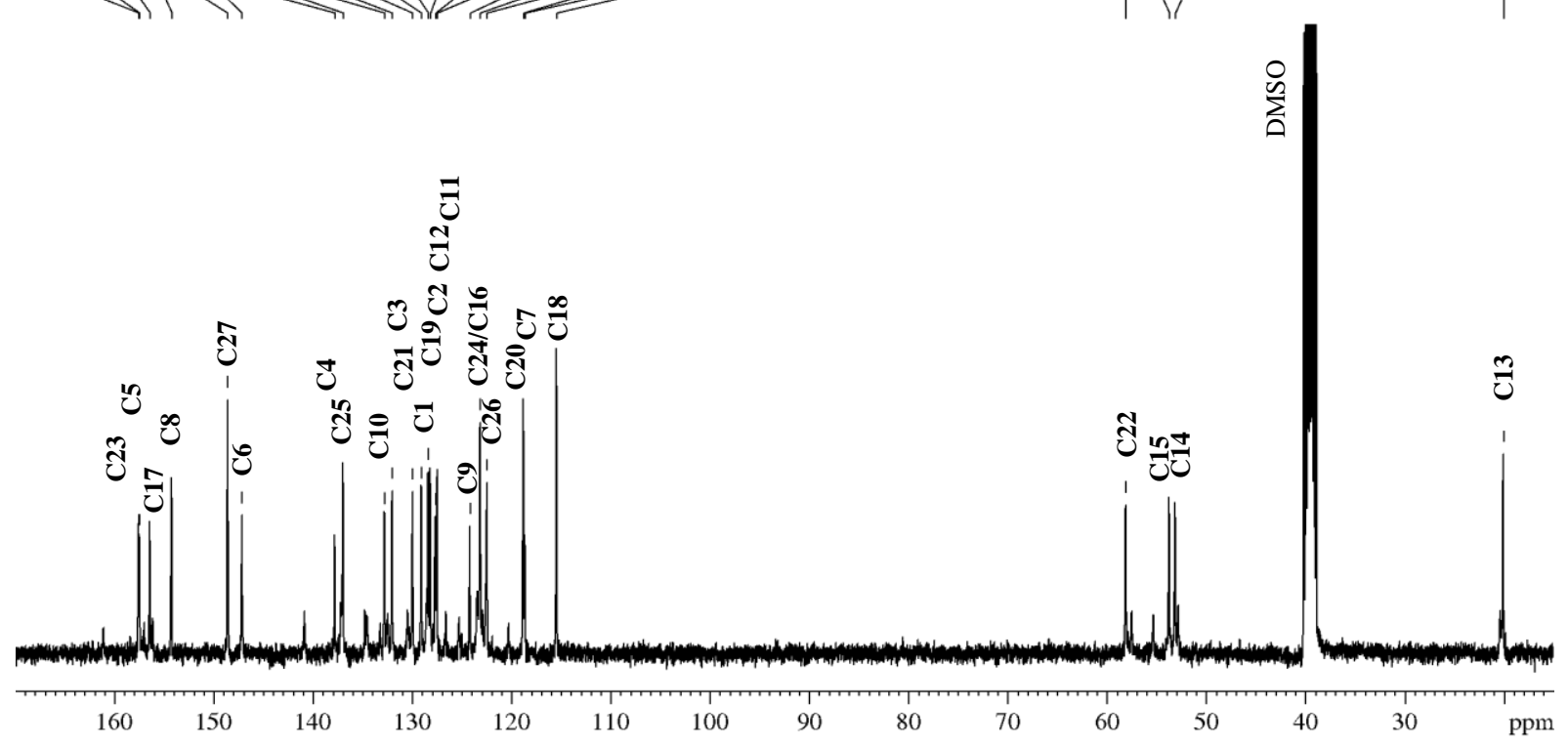

Figure S7. ${ }^{13} \mathrm{C}$ NMR spectra $(100 \mathrm{MHz})$ of $\mathbf{H}_{3} \mathbf{L} \mathbf{1}$ in DMSO- $d_{6}$ at room temperature.

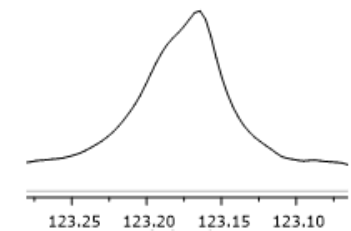

$\stackrel{\circ}{\sum^{n}}$

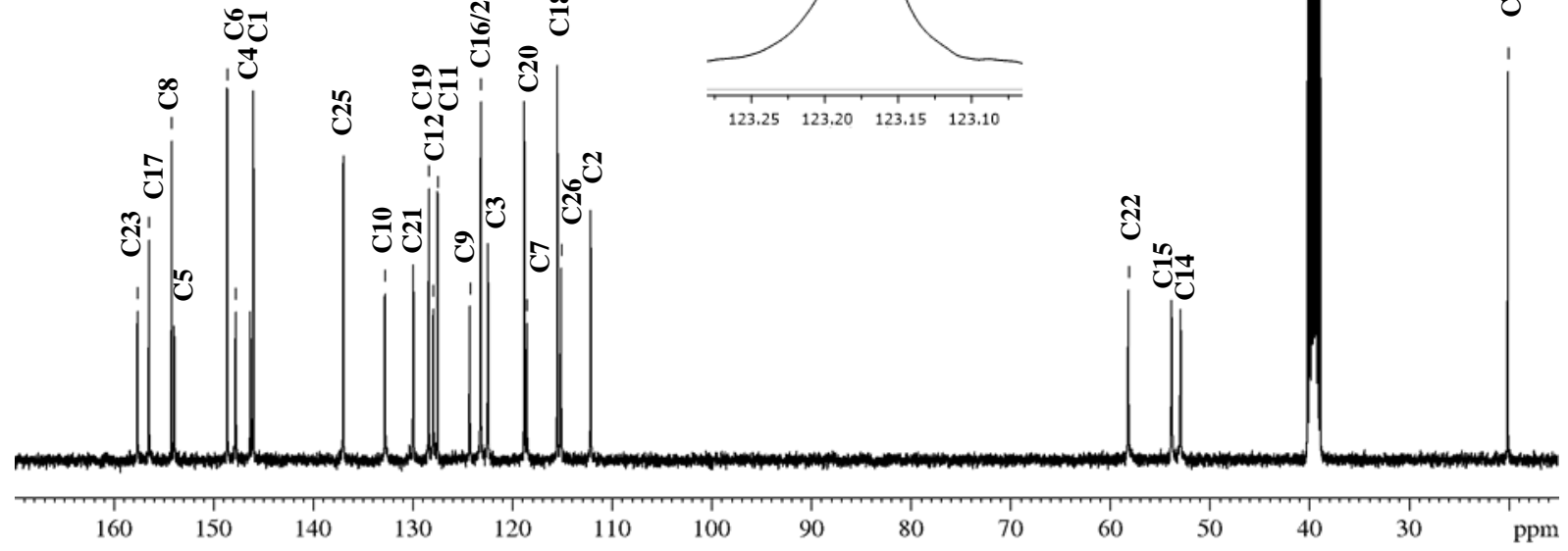

Figure S8. ${ }^{13} \mathrm{C}$ NMR spectra $(100 \mathrm{MHz})$ of $\mathbf{H}_{3} \mathbf{L} \mathbf{2}$ in DMSO- $d_{6}$ at room temperature. 
Table S1. ${ }^{1} \mathrm{H}(400 \mathrm{MHz})$ and ${ }^{13} \mathrm{C}(100 \mathrm{MHz})$ data for $\mathbf{H}_{3} \mathbf{L} \mathbf{1}$ and $\mathbf{H 3 L 2}$ in DMSO- $d_{6}$ at room temperature, along with the respective assignments. Chemical shifts $(\delta)$ are in ppm and coupling constants $(J)$ are in $\mathrm{Hz}$.

\begin{tabular}{|c|c|c|c|c|}
\hline \multirow[b]{2}{*}{$\mathbf{C} / \mathbf{H}$} & \multicolumn{2}{|r|}{$\mathbf{H}_{3} \mathbf{L} \mathbf{1}$} & \multicolumn{2}{|r|}{$\mathrm{H}_{3} \mathrm{L2}$} \\
\hline & $\delta \mathrm{c}$ & $\delta_{\mathbf{H}}$ & $\delta \mathrm{c}$ & $\delta_{H}$ \\
\hline 1 & 129.10 & $7.94\left(\mathrm{~d}, 1 \mathrm{H},{ }^{3} J_{\mathrm{HH}}=4.5\right)$ & 146.02 & $7.96\left(\mathrm{~d}, 1 \mathrm{H},{ }^{3} J_{\mathrm{HH}}=1.4\right)$ \\
\hline 2 & 128.21 & $7.23\left(\mathrm{t}, 1 \mathrm{H},{ }^{3} J_{\mathrm{HH}}=4.5\right)$ & 112.15 & $6.70\left(\mathrm{dd}, 1 \mathrm{H},{ }^{3} J_{\mathrm{HH}}=3.4,{ }^{3} J_{\mathrm{HH}}=1.4\right)$ \\
\hline 3 & 132.04 & $7.88\left(\mathrm{~d}, 1 \mathrm{H},{ }^{3} J_{\mathrm{HH}}=4.5\right)$ & 122.46 & $7.33-7.30(\mathrm{~m}, 2 \mathrm{H})^{*}$ \\
\hline 4 & 137.83 & - & 146.32 & - \\
\hline 5 & 157.48 & - & 153.96 & - \\
\hline 6 & 147.17 & $8.63(\mathrm{~s}, 1 \mathrm{H})$ & 147.79 & $8.64(\mathrm{~s}, 1 \mathrm{H})$ \\
\hline 7 & 118.67 & - & 118.55 & - \\
\hline 8 & 154.27 & - & 154.23 & - \\
\hline 9 & 124.21 & - & 124.29 & - \\
\hline 10 & 132.80 & $7.12(\mathrm{~s}, 1 \mathrm{H})$ & 132.80 & $7.12(\mathrm{~s}, 1 \mathrm{H})$ \\
\hline 11 & 127.51 & - & 127.50 & - \\
\hline 12 & 127.70 & $7.32-7.30(\mathrm{~m}, 2 \mathrm{H})^{*}$ & 127.95 & $7.27(\mathrm{~s}, 1 \mathrm{H})$ \\
\hline 13 & 20.07 & $2.22(\mathrm{~s}, 3 \mathrm{H})$ & 20.05 & $2.21(\mathrm{~s}, 3 \mathrm{H})$ \\
\hline 14 & 53.14 & $3.71(\mathrm{~s}, 2 \mathrm{H})$ & 52.92 & $3.70(\mathrm{~s}, 2 \mathrm{H})$ \\
\hline 15 & 53.74 & $3.68(\mathrm{~s}, 2 \mathrm{H})$ & 53.82 & $3.69(\mathrm{~s}, 2 \mathrm{H})$ \\
\hline 16 & 123.16 & - & $123.17 * *$ & - \\
\hline 17 & 156.48 & - & 156.50 & - \\
\hline 18 & 115.48 & $6.77\left(\mathrm{~d}, 1 \mathrm{H},{ }^{3} J_{\mathrm{HH}}=8.0\right)$ & 115.49 & $6.77\left(\mathrm{~d}, 1 \mathrm{H},{ }^{3} J_{\mathrm{HH}}=7.4\right)$ \\
\hline 19 & 128.41 & $7.09\left(\mathrm{td}, 1 \mathrm{H},{ }^{3} J_{\mathrm{HH}}=7.7,{ }^{4} J_{\mathrm{HH}}=1.5\right)$ & 128.40 & $7.09\left(\mathrm{td}, 1 \mathrm{H},{ }^{3} J_{\mathrm{HH}}=7.4,{ }^{4} J_{\mathrm{HH}}=1.0\right)$ \\
\hline 20 & 118.82 & $6.75\left(\mathrm{t}, 1 \mathrm{H},{ }^{3} J_{\mathrm{HH}}=7.4\right)$ & 118.80 & $6.75\left(\mathrm{t}, 1 \mathrm{H},{ }^{3} J_{\mathrm{HH}}=7.4\right)$ \\
\hline 21 & 129.97 & $7.20\left(\mathrm{dd}, 1 \mathrm{H},{ }^{3} J_{\mathrm{HH}}=7.4,{ }^{4} J_{\mathrm{HH}}=1.0\right)$ & 129.95 & $7.20\left(\mathrm{dd}, 1 \mathrm{H},{ }^{3} J_{\mathrm{HH}}=7.4,{ }^{4} J_{\mathrm{HH}}=1.0\right)$ \\
\hline 22 & 58.10 & $3.79(\mathrm{~s}, 2 \mathrm{H})$ & 58.16 & $3.78(\mathrm{~s}, 2 \mathrm{H})$ \\
\hline 23 & 157.60 & - & 157.65 & - \\
\hline 24 & 123.20 & $7.41\left(\mathrm{~d}, 1 \mathrm{H},{ }^{3} J_{\mathrm{HH}}=7.4\right)$ & $123.17 * *$ & $7.40\left(\mathrm{~d}, 1 \mathrm{H},{ }^{3} J_{\mathrm{HH}}=7.8\right)$ \\
\hline 25 & 137.00 & $7.80\left(\mathrm{t}, 1 \mathrm{H},{ }^{3} J_{\mathrm{HH}}=7.4\right)$ & 136.97 & $7.78\left(\mathrm{td}, 1 \mathrm{H},{ }^{3} J_{\mathrm{HH}}=7.8,{ }^{4} J_{\mathrm{HH}}=1.6\right)$ \\
\hline 26 & 122.48 & $7.32-7.30(\mathrm{~m}, 2 \mathrm{H})^{*}$ & 115.12 & $7.33-7.30(\mathrm{~m}, 2 \mathrm{H})^{*}$ \\
\hline 27 & 148.61 & $8.56\left(\mathrm{~d}, 1 \mathrm{H},{ }^{3} J_{\mathrm{HH}}=3.9\right)$ & 148.64 & $8.56\left(\mathrm{~d}, 1 \mathrm{H},{ }^{3} J_{\mathrm{HH}}=4.1\right)$ \\
\hline $\mathrm{O} 2-\mathrm{H}$ & - & $12.05(\mathrm{~s}, 1 \mathrm{H})$ & - & $12.06(\mathrm{~s}, 1 \mathrm{H})$ \\
\hline
\end{tabular}

*Overlapped signals. **Unresolved asymmetric signal (see Inset of Figure S8). Signal multiplicity s: singlet, d: doublet, t: triplet, dd: doublet of doublets, td: triplet of doublets. 


\section{Structural properties of the complexes.}

Table S2. Experimental details for complex 1.

\begin{tabular}{|c|c|}
\hline Crystal & Complex 1 \\
\hline Chemical formula & $\mathrm{C}_{27} \mathrm{H}_{25} \mathrm{ClCu}_{2} \mathrm{~N}_{4} \mathrm{O}_{8} \mathrm{~S}$ \\
\hline $\operatorname{Mr}\left(\mathrm{g} \mathrm{mol}^{-1}\right)$ & 728.10 \\
\hline Crystal system, space group & Monoclinic, $P 21 / c$ \\
\hline Temperature (K) & 293 \\
\hline$a, b, c(\AA)$ & $10.8166(1), 21.0642(2), 14.6498(2)$ \\
\hline$\beta\left(^{\circ}\right)$ & $106.415(1)$ \\
\hline$V\left(\AA^{3}\right)$ & $3201.80(6)$ \\
\hline$Z$ & 4 \\
\hline Radiation type & Mo $K \alpha$ \\
\hline$\mu\left(\mathrm{mm}^{-1}\right)$ & 1.528 \\
\hline Crystal size $(\mathrm{mm})$ & $0.41 \times 0.36 \times 0.23$ \\
\hline \multicolumn{2}{|l|}{ Data collection } \\
\hline Diffractometer & SuperNova, Dual, Atlas S2 \\
\hline No. of measured, independent and observed & $68355,8419,7522$ \\
\hline$R_{\text {int }}$ & 0.011 \\
\hline \multicolumn{2}{|l|}{ Refinement } \\
\hline$R\left[F^{2}>2 \sigma\left(F^{2}\right)\right], w R\left(F^{2}\right), \mathrm{S}$ & $0.034,0.103,1.06$ \\
\hline No. of parameters & 417 \\
\hline No. of restraints & 0 \\
\hline$\Delta \rho \max , \Delta \rho \min (\mathrm{e} \AA$ - 3 ) & $1.55,-0.70$ \\
\hline
\end{tabular}

Table S3. H-bonding parameters for complex $1\left(\AA{ }^{\circ}\right)$.

\begin{tabular}{lllll}
\hline $\mathrm{D}-\mathrm{H} \cdots \mathrm{A}$ & $\mathrm{D}-\mathrm{H}$ & $\mathrm{H} \cdots \mathrm{A}$ & $\mathrm{D} \cdots \mathrm{A}$ & $\mathrm{D}-\mathrm{H} \cdots \mathrm{A}$ \\
\hline $\mathrm{O} 2-\mathrm{H} 2 \mathrm{~A} \cdots \mathrm{O} 6^{i i}$ & 0.82 & 2.170 & $3.038(15)$ & 177.0 \\
$\mathrm{O} 4-\mathrm{H} 4 \cdots \mathrm{O}^{i i}$ & 0.82 & 1.940 & $2.740(9)$ & 166.0 \\
$\mathrm{C} 23-\mathrm{H} 23 \mathrm{~A} \cdots \mathrm{O}^{i}$ & 0.97 & 2.460 & $3.206(3)$ & 134.0 \\
$\mathrm{C} 23-\mathrm{H} 23 \mathrm{~B} \cdots$ O $^{\mathrm{iii}}$ & 0.97 & 2.330 & $3.252(5)$ & 158.0 \\
\hline Symmetry codes: $(\boldsymbol{i}) 1-x,-y, 2-z ;(\boldsymbol{i i}) x, y, l+z ;(\boldsymbol{i i i}) l-x,-1 / 2+y, 1+1 / 2-z$. &
\end{tabular}


Table S4. Experimental details for complex 2.

\begin{tabular}{|c|c|}
\hline Crystal & Complex 2 \\
\hline Chemical formula & $\mathrm{C}_{27} \mathrm{H}_{25} \mathrm{ClCu}_{2} \mathrm{~N}_{4} \mathrm{O}_{9}$ \\
\hline$M \mathrm{r}\left(\mathrm{g} \mathrm{mol}^{-1}\right)$ & 712.04 \\
\hline Crystal system, space group & Monoclinic, $P 21 / c$ \\
\hline Temperature (K) & 294 \\
\hline$a, b, c(\AA)$ & $10.5312(5), 14.8760(7), 18.8006(9)$ \\
\hline$\beta\left(^{\circ}\right)$ & $91.967(5)$ \\
\hline$V\left(\AA^{3}\right)$ & $2943.6(2)$ \\
\hline$Z$ & 4 \\
\hline Radiation type & Mo $K \alpha$ \\
\hline$\mu\left(\mathrm{mm}^{-1}\right)$ & 1.595 \\
\hline Crystal size $(\mathrm{mm})$ & $0.17 \times 0.25 \times 0.65$ \\
\hline \multicolumn{2}{|l|}{ Data collection } \\
\hline Diffractometer & SuperNova, Dual, Atlas S2 \\
\hline No. of measured, independent and observed & $81145,7904,5908$ \\
\hline$R_{\text {int }}$ & 0.0845 \\
\hline \multicolumn{2}{|l|}{ Refinement } \\
\hline$R\left[F^{2}>2 \sigma\left(F^{2}\right)\right], w R\left(F^{2}\right), \mathrm{S}$ & $0.0522,0.1185,1.031$ \\
\hline No. of parameters & 391 \\
\hline No. of restraints & 0 \\
\hline$\Delta \rho \max , \Delta \rho \min (\mathrm{e} \AA$ \&-3) & $0.707,-0.595$ \\
\hline
\end{tabular}

Table S5. H-bonding parameters for complex $2\left(\AA{ }^{\circ}\right)$.

\begin{tabular}{|c|c|c|c|c|}
\hline $\mathrm{D}-\mathrm{H} \cdots \mathrm{A}$ & $\mathrm{D}-\mathrm{H}$ & $\mathrm{H} \cdots \mathrm{A}$ & $\mathrm{D} \cdots \mathrm{A}$ & $\mathrm{D}-\mathrm{H} \cdots \mathrm{A}$ \\
\hline $\mathrm{O} 2-\mathrm{H} 2 \mathrm{~A} \cdots \mathrm{O} 5$ & 0.82 & 2.120 & $2.940 \quad(4)$ & 173.0 \\
\hline $\mathrm{O} 4-\mathrm{H} 4 \cdots \mathrm{O} 6$ & 0.82 & 1.940 & $2.758 \quad(4)$ & 176.0 \\
\hline $\mathrm{C} 22-\mathrm{H} 22 \mathrm{~A} \cdots \mathrm{O} 1^{i}$ & 0.97 & 2.360 & $3.097 \quad$ (4) & 132.0 \\
\hline
\end{tabular}

Symmetry code: $(\boldsymbol{i})-x+1,-y+1,-z+1$. 
Vibrational spectroscopy characterization of the compounds.

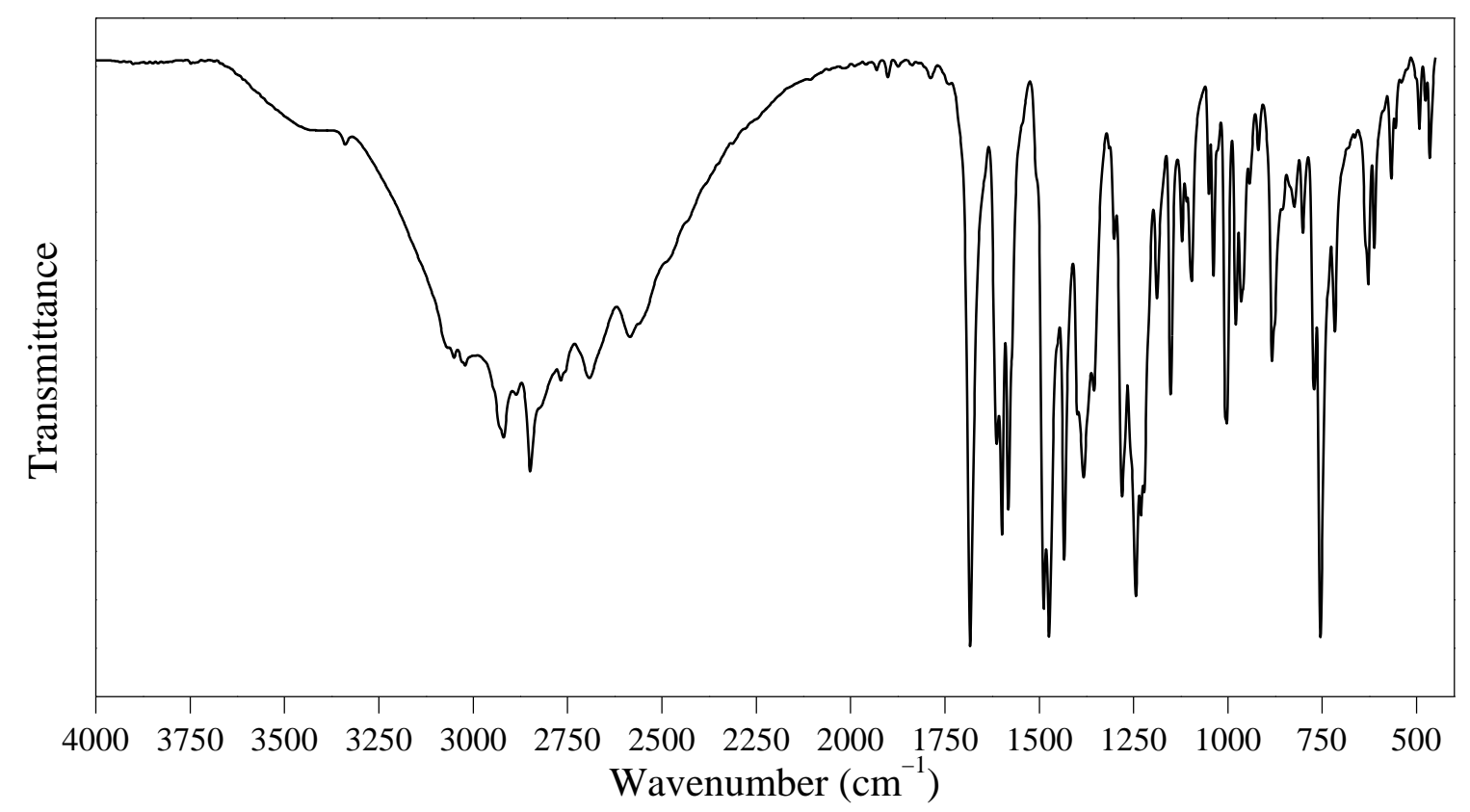

Figure S9. FTIR spectra of the HBPAMFF precursor in $\mathrm{KBr}$ pellets. 

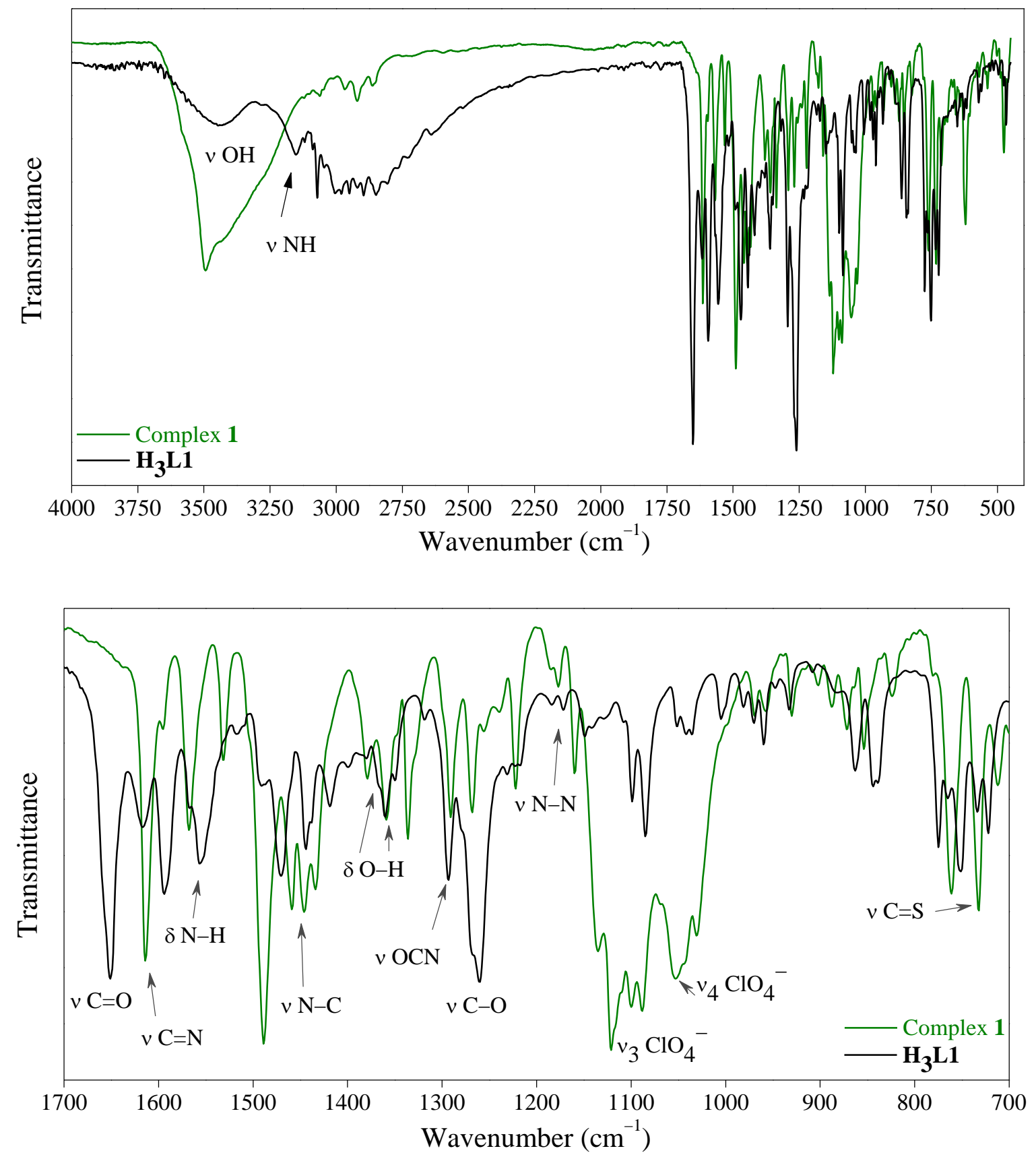

Figure S10. Top. FTIR spectra of $\mathbf{H}_{3} \mathbf{L} 1$ and Complex 1 in $\mathrm{KBr}$ pellets. Bottom. Detail of the $1700-700 \mathrm{~cm}^{-1}$ region. In both cases, the main assignments are indicated. 

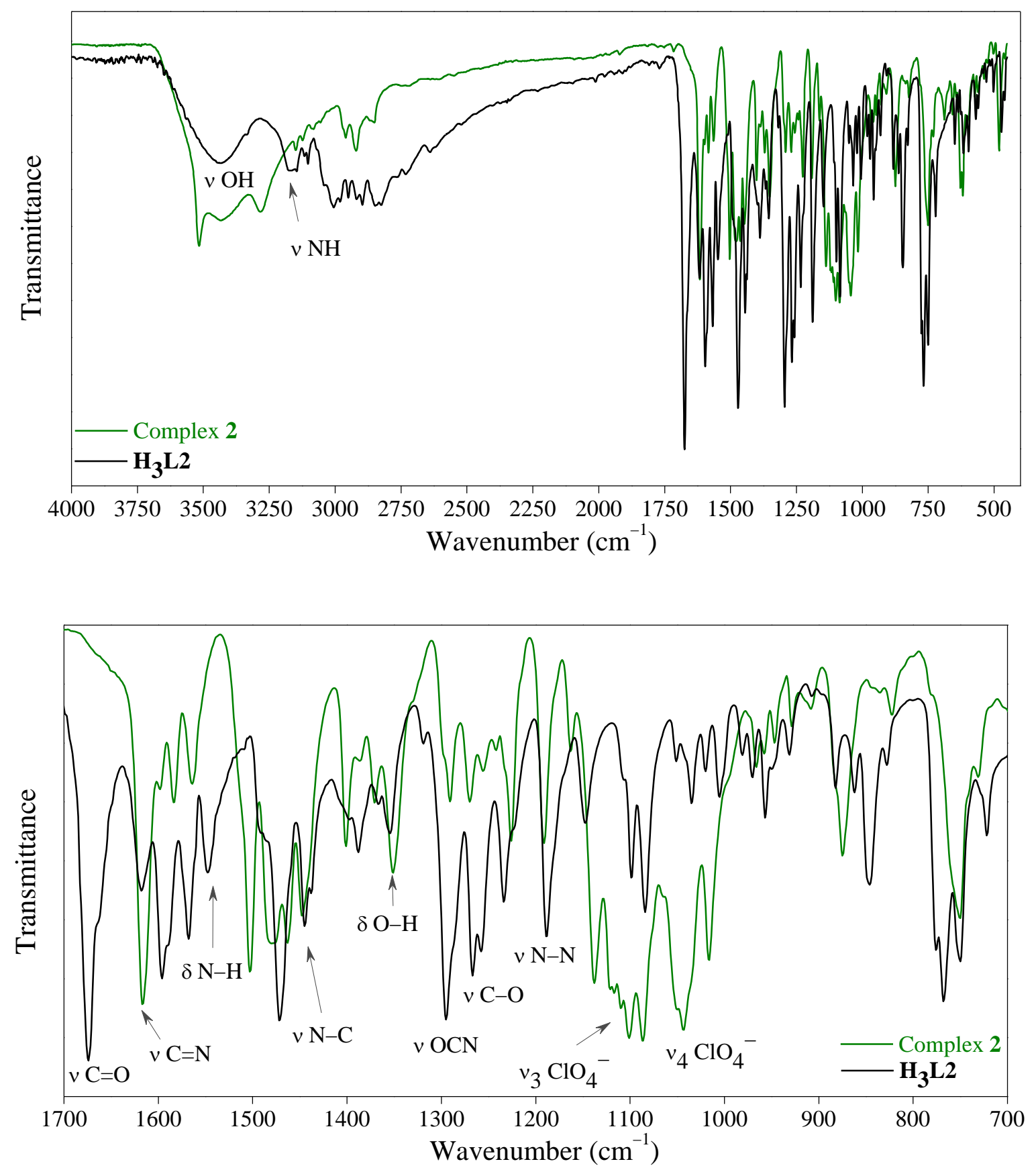

Figure S11. Top. FTIR spectra of $\mathbf{H}_{3} \mathbf{L} 2$ and Complex $\mathbf{2}$ in $\mathrm{KBr}$ pellets. Bottom. Detail of the $1700-700 \mathrm{~cm}^{-1}$ region. In both cases, the main assignments are indicated. 


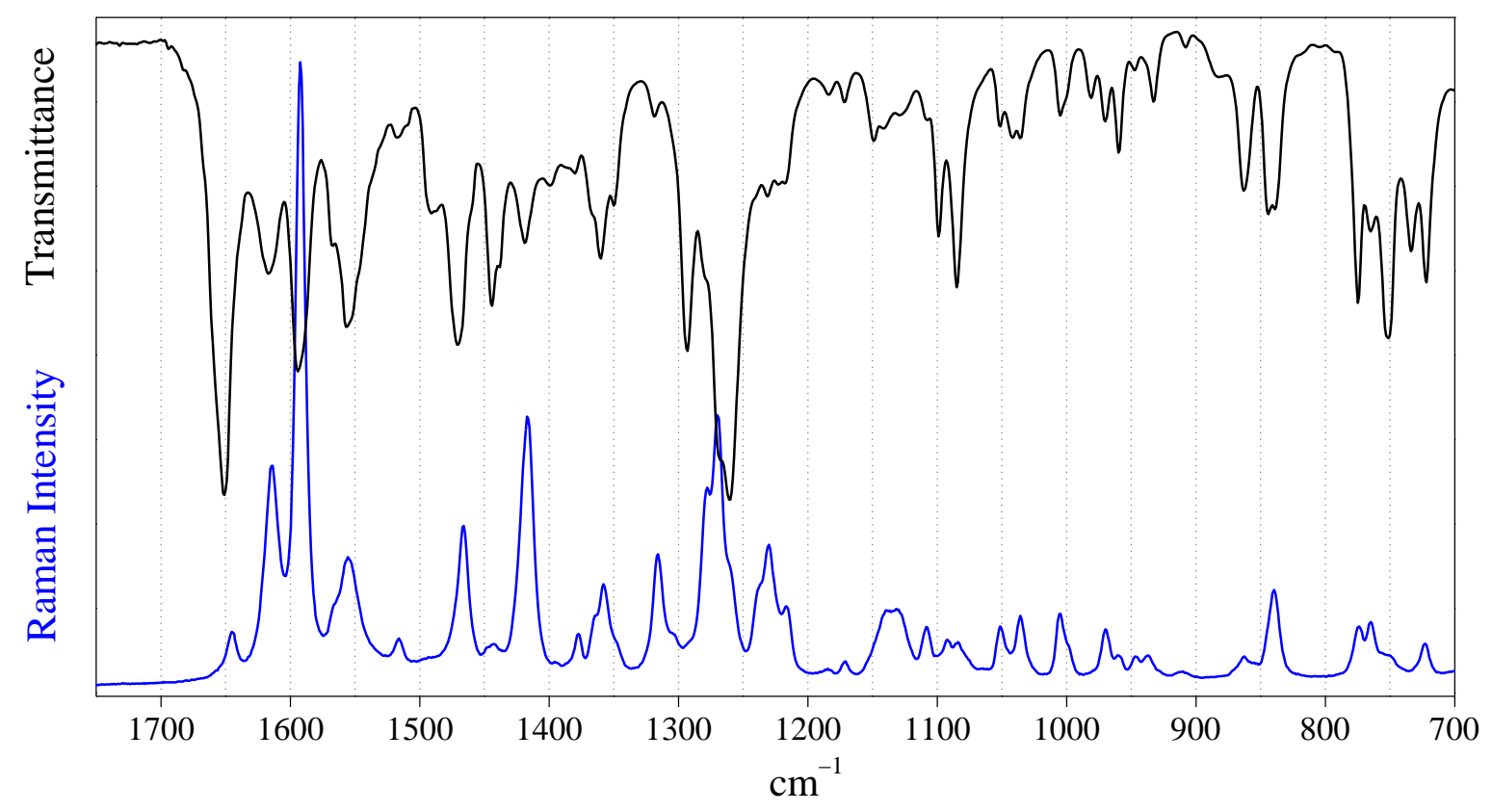

Figure S12. FTIR and Raman spectra of $\mathbf{H}_{3} \mathbf{L} \mathbf{1}$ in the region $1700-700 \mathrm{~cm}^{-1}$.

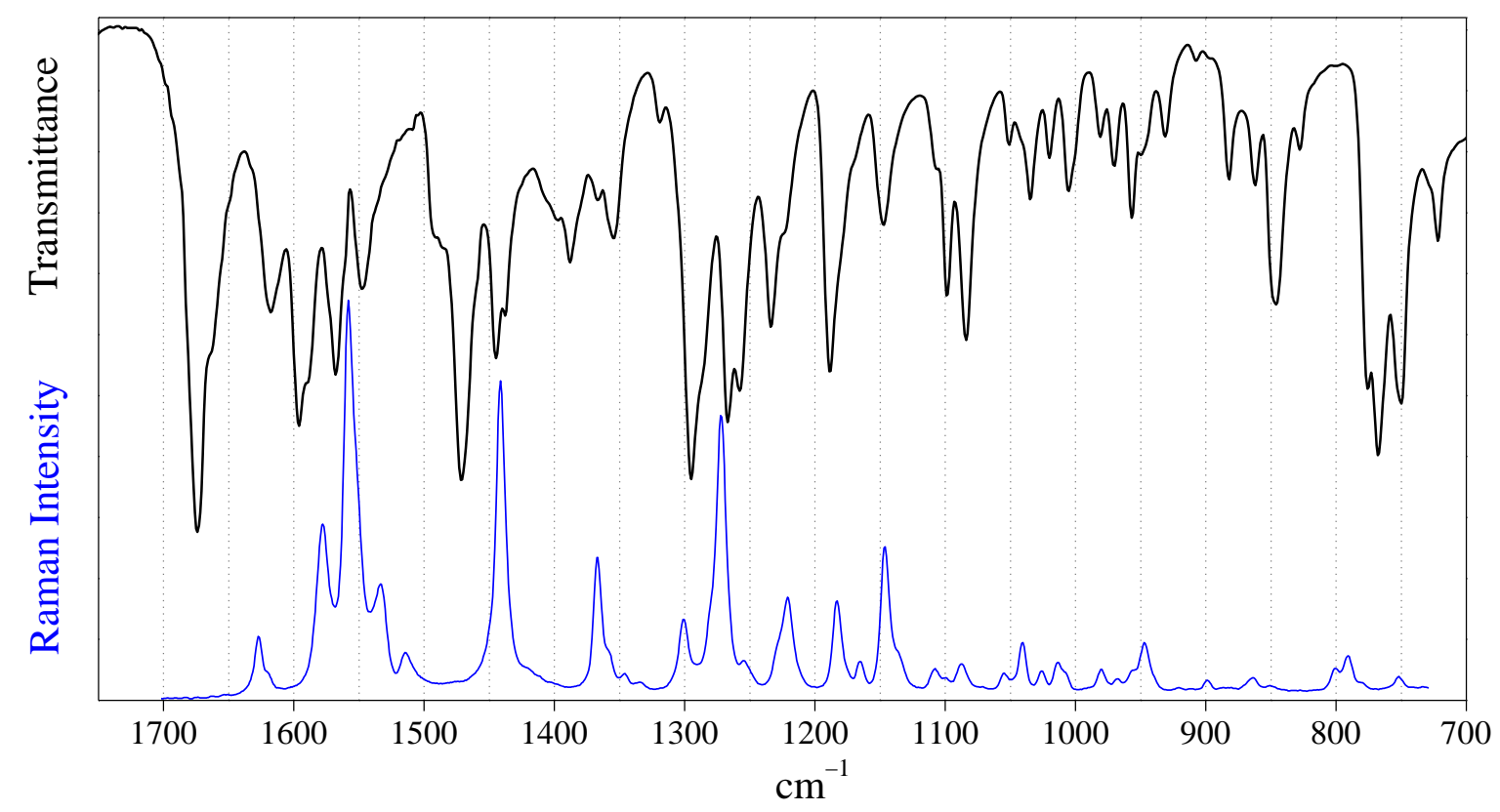

Figure S13. FTIR and Raman spectra of $\mathbf{H}_{3} \mathbf{L} 2$ in the region $1700-700 \mathrm{~cm}^{-1}$. 


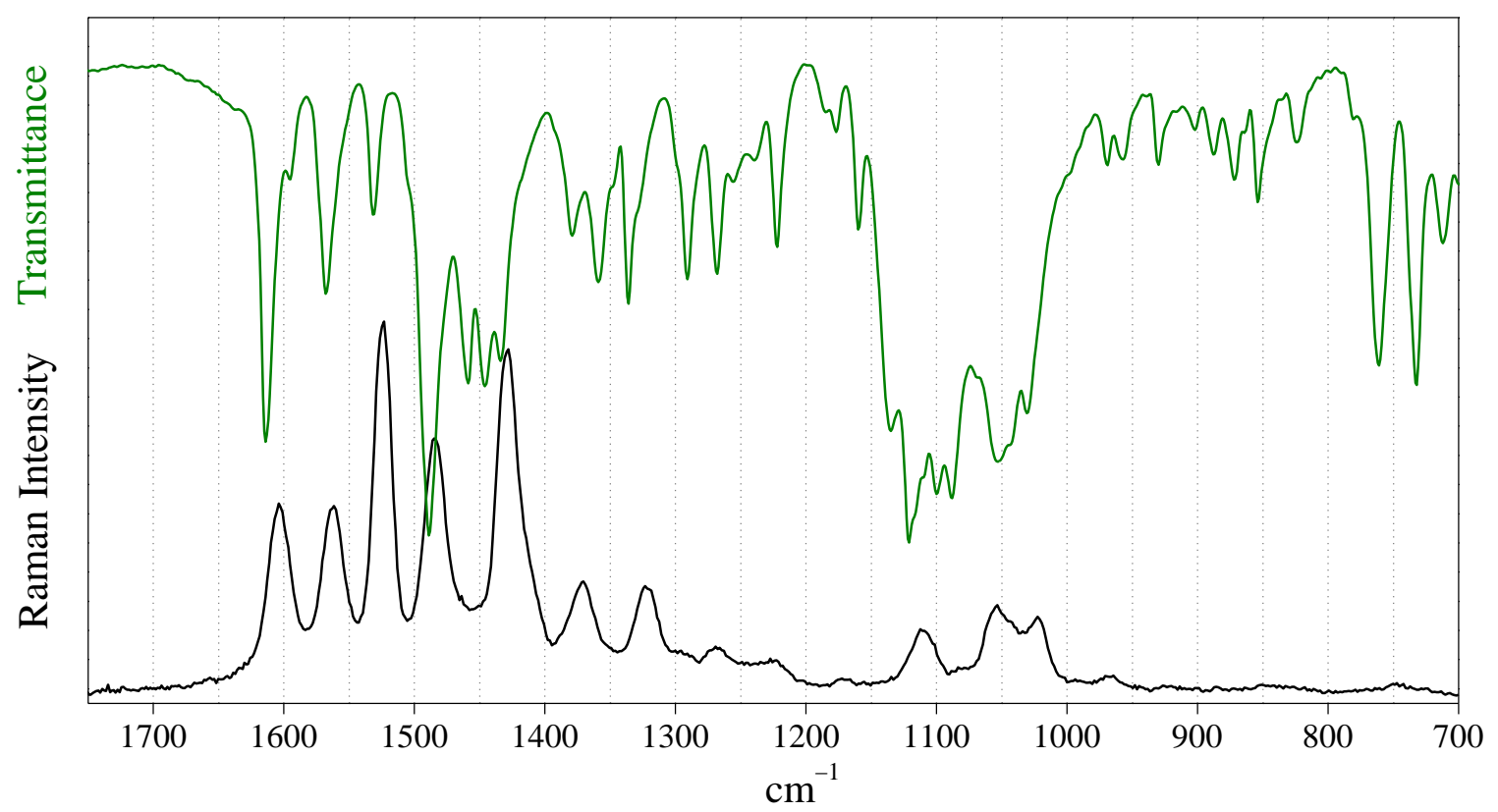

Figure S14. FTIR and Raman spectra of complex 1 in the region $1700-700 \mathrm{~cm}^{-1}$.

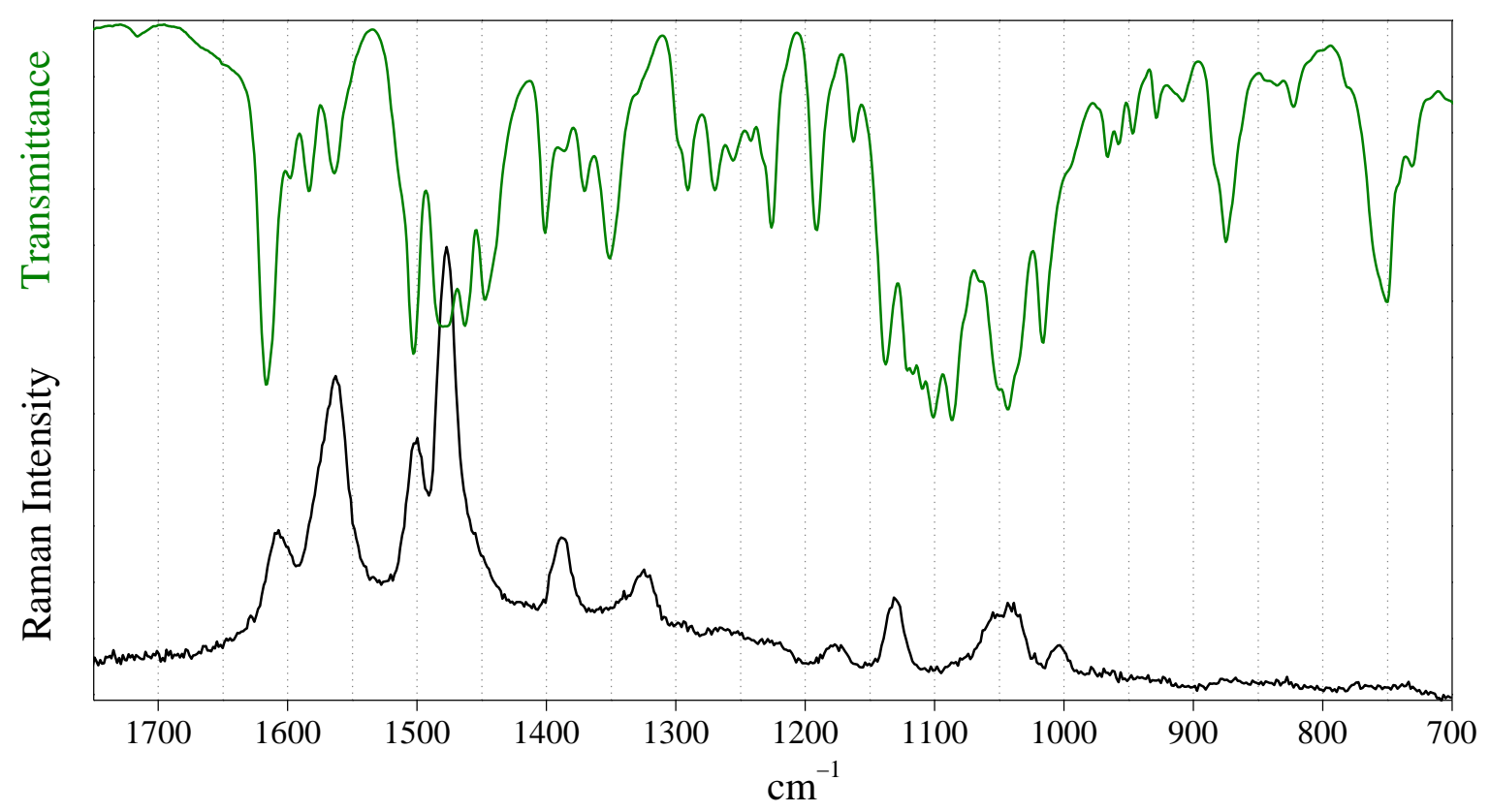

Figure S15. FTIR and Raman spectra of complex 2 in the region $1700-700 \mathrm{~cm}^{-1}$. 
Table S6. Selected vibrational absorptions $\left(\mathrm{cm}^{-1}\right)$ for $\mathbf{H}_{3} \mathbf{L} \mathbf{1}$ and $\mathbf{H}_{3} \mathbf{L} 2$ and their complexes.

\begin{tabular}{cc|cc|cc|cc|c}
\hline \multicolumn{2}{c|}{ H3L1 $_{3}$} & \multicolumn{2}{c|}{ H3L2 $_{3}$} & \multicolumn{2}{c|}{$\mathbf{1}$} & \multicolumn{2}{c|}{$\mathbf{2}$} & Assignment \\
\hline IR & Raman & IR & Raman & IR & Raman & IR & Raman & \\
3446 & - & 3440 & - & 3490 & - & 3432 & - & $v$ OH \\
3151 & - & 3149 & - & - & - & - & - & $v$ NH \\
1651 & 1645 & 1674 & 1668 & 1488 & 1485 & 1502 & 1501 & $v \mathrm{C}=\mathrm{O}$ \\
1618 & 1614 & 1616 & 1608 & 1614 & 1615 & 1616 & 1607 & $\mathrm{C}=\mathrm{N}$ \\
1555 & 1556 & 1545 & 1546 & - & - & - & - & $\delta \mathrm{N}-\mathrm{H}$ \\
$1366(\mathrm{sh})$ & $1365(\mathrm{sh})$ & $1358(\mathrm{sh})$ & 1365 & - & - & - & - & $\delta$ O-H phenol\#1 \\
1360 & 1358 & 1355 & 1352 & 1359 & - & 1351 & - & $\delta$ O-H phenol\#2 \\
1172 & 1171 & $1169(\mathrm{sh})$ & 1171 & 1177 & 1175 & $1179(\mathrm{sh})$ & 1177 & $v$ N-N \\
- & - & - & - & 1120 & - & 1118 & - & $v_{3} \mathrm{ClO}_{4}^{-}$ \\
- & - & - & - & 1052 & 1053 & 1045 & 1043 & $v_{4} \mathrm{ClO}_{4}^{-}$ \\
\hline \multicolumn{7}{c}{ sh: shoulder. }
\end{tabular}

Table S7. UV-Vis absorption properties of $\mathrm{H}_{3} \mathrm{LL}$ and $\mathrm{H}_{3} \mathrm{~L} 2$, as well as those of their dicopper(II) complexes 1 and 2, in DMSO at r.t. Suggested assignments are also included.

\begin{tabular}{|c|c|c|c|c|}
\hline \multicolumn{2}{|r|}{$\mathrm{H}_{3} \mathrm{~L} \mathbf{1}$} & \multicolumn{2}{|c|}{$\mathrm{H}_{3} \mathrm{~L} 2$} & \multirow[t]{2}{*}{ Assignment } \\
\hline$\lambda(\mathrm{nm})$ & $\varepsilon\left(\mathrm{M}^{-1} \mathrm{~cm}^{-1}\right)$ & $\lambda(\mathrm{nm})$ & $\varepsilon\left(\mathrm{M}^{-1} \mathrm{~cm}^{-1}\right)$ & \\
\hline 344 & 18500 & 343 & 18700 & HBPAMFF rings \\
\hline 312 & 18900 & 310 & 24000 & $\mathrm{CO}-\mathrm{NH}-\mathrm{N}=\mathrm{N}$ \\
\hline 300 & 18300 & 298 & 23500 & $\mathrm{CO}-\mathrm{NH}-\mathrm{N}=\mathrm{N}$ \\
\hline 286 & 15100 & 287 & 18000 & Furan / thiophene and \\
\hline 259 & 15000 & 258 & 14100 & HBPAMFF rings \\
\hline \multicolumn{2}{|r|}{1} & \multicolumn{2}{|c|}{2} & Assignment \\
\hline$\lambda(\mathrm{nm})$ & $\varepsilon\left(\mathrm{M}^{-1} \mathrm{~cm}^{-1}\right)$ & $\lambda(\mathrm{nm})$ & $\varepsilon\left(\mathrm{M}^{-1} \mathrm{~cm}^{-1}\right)$ & \\
\hline 667 & 180 & 667 & 150 & $d-d$ transitions \\
\hline 400 & 14730 & 397 & 13200 & HBPAMFF rings \\
\hline 343 & 14500 & 340 & 15000 & $\mathrm{CO}-\mathrm{NH}-\mathrm{N}=\mathrm{N}$ \\
\hline 329 & 14100 & 325 & 14500 & $\mathrm{CO}-\mathrm{NH}-\mathrm{N}=\mathrm{N}$ \\
\hline 313 & 10900 & 310 & 11300 & HBPAMFF rings \\
\hline $281(\mathrm{sh})$ & 16800 & 287 & 14300 & Furan / thiophene and \\
\hline 264 & 18000 & 265 & 17800 & HBPAMFF rings \\
\hline
\end{tabular}




\section{UV-Visible stability studies.}
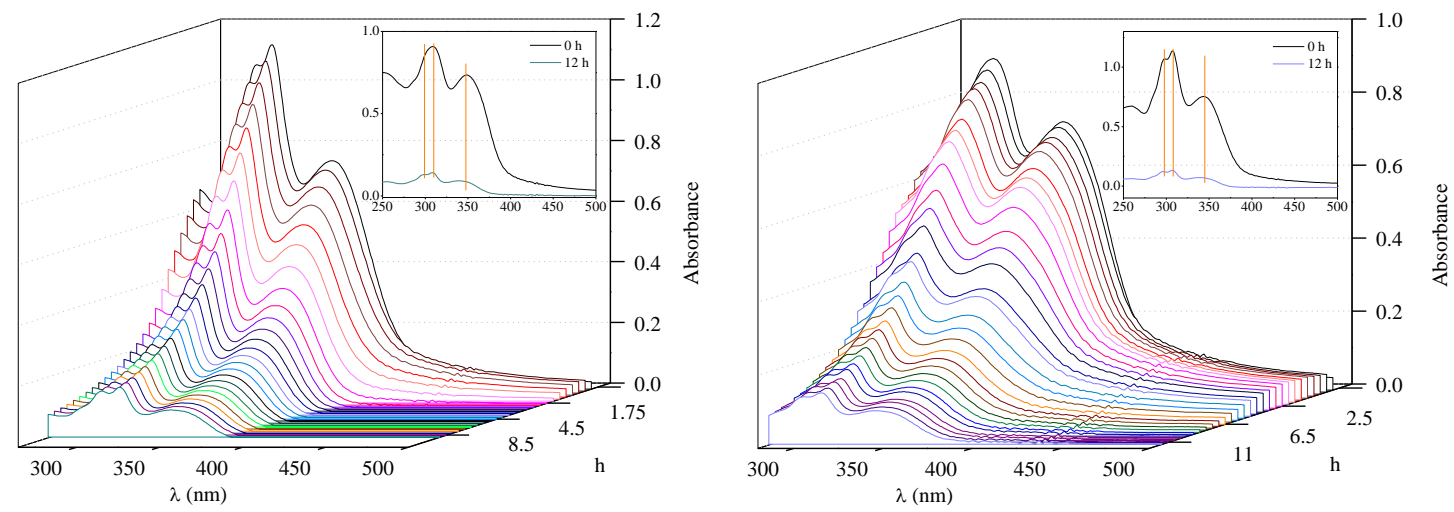

Figure S16. UV-Vis spectra of $\mathbf{H}_{3} \mathbf{L} \mathbf{1}$ (left, $\mathrm{pH}$ 6.15) and $\mathbf{H}_{3} \mathbf{L} 2$ (right, $\mathrm{pH}$ 6.35) vs time in $10 \%$ DMSO at r.t. $[\mathrm{L}]=5 \times 10^{-5} \mathrm{M}$. Inset: Comparison between spectra at 0 and $12 \mathrm{~h}$.
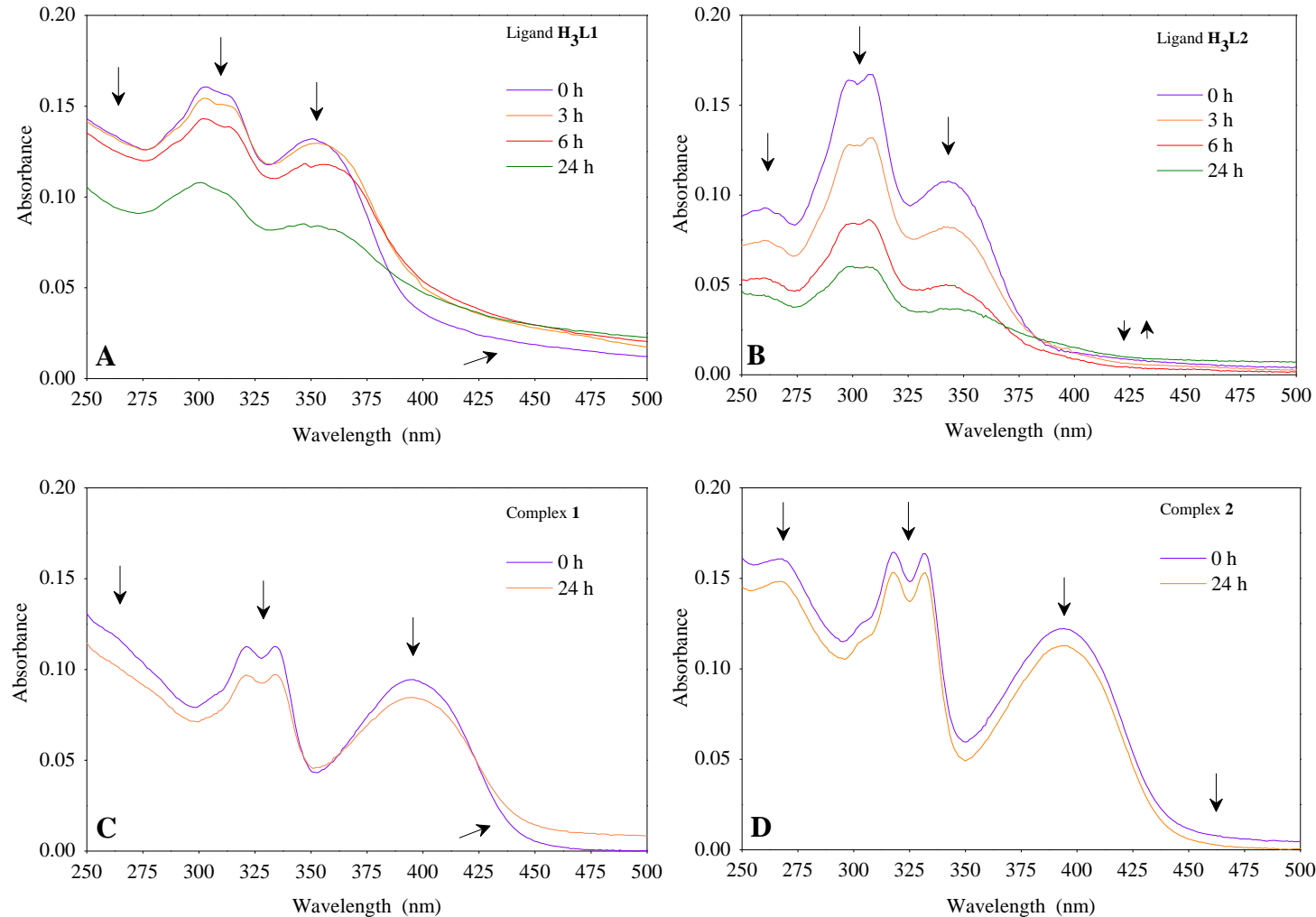

Figure S17. UV-Vis spectra over time (24 h) for the ligands $\mathbf{H}_{3} \mathbf{L} 1$ (A) and $\mathbf{H}_{3} \mathbf{L} 2$ (B), and complexes 1 (C) and 2 (D) in 10\% DMSO-90\% PBS (pH 7.4) at r.t. [ ] = $1 \times 10^{-5} \mathrm{M}$. 

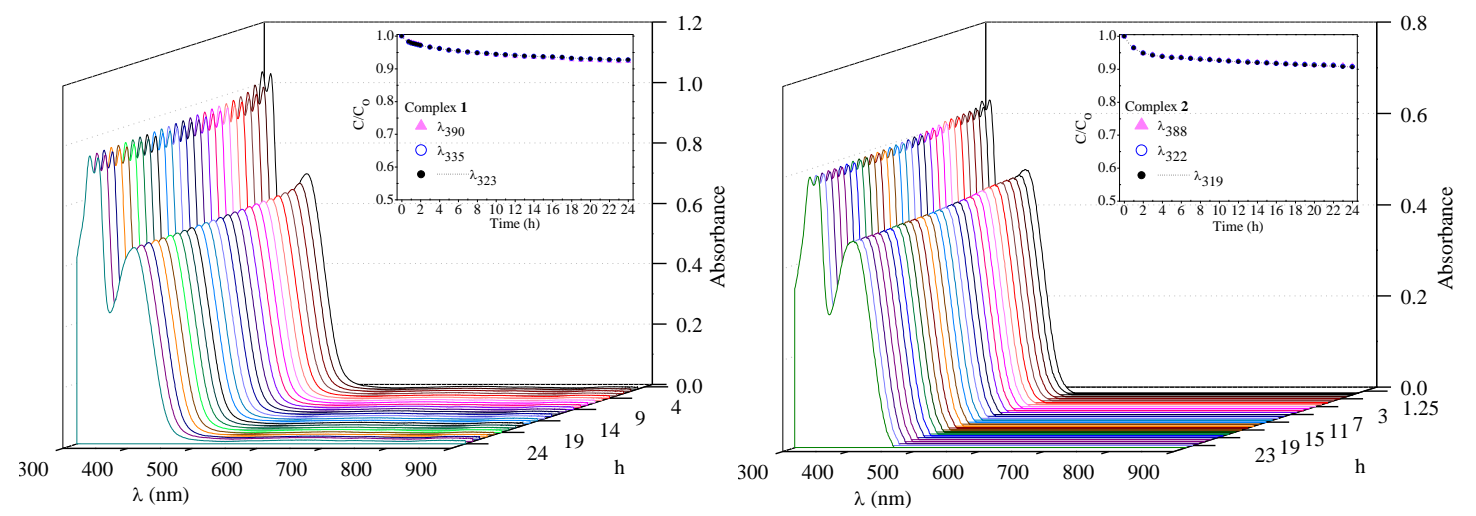

Figure S18. UV-Visible spectra of 1 (left) and 2 (right), along 24 h, in $10 \%$ DMSO at r.t. $[\mathrm{C}]=5 \times 10^{-5} \mathrm{M} . \mathrm{pH}$ 6.0. Inset: $\mathrm{C} / \mathrm{C}_{\mathrm{o}}$ versus time at different wavelengths. 


\section{ESI-MS(+) analyses.}
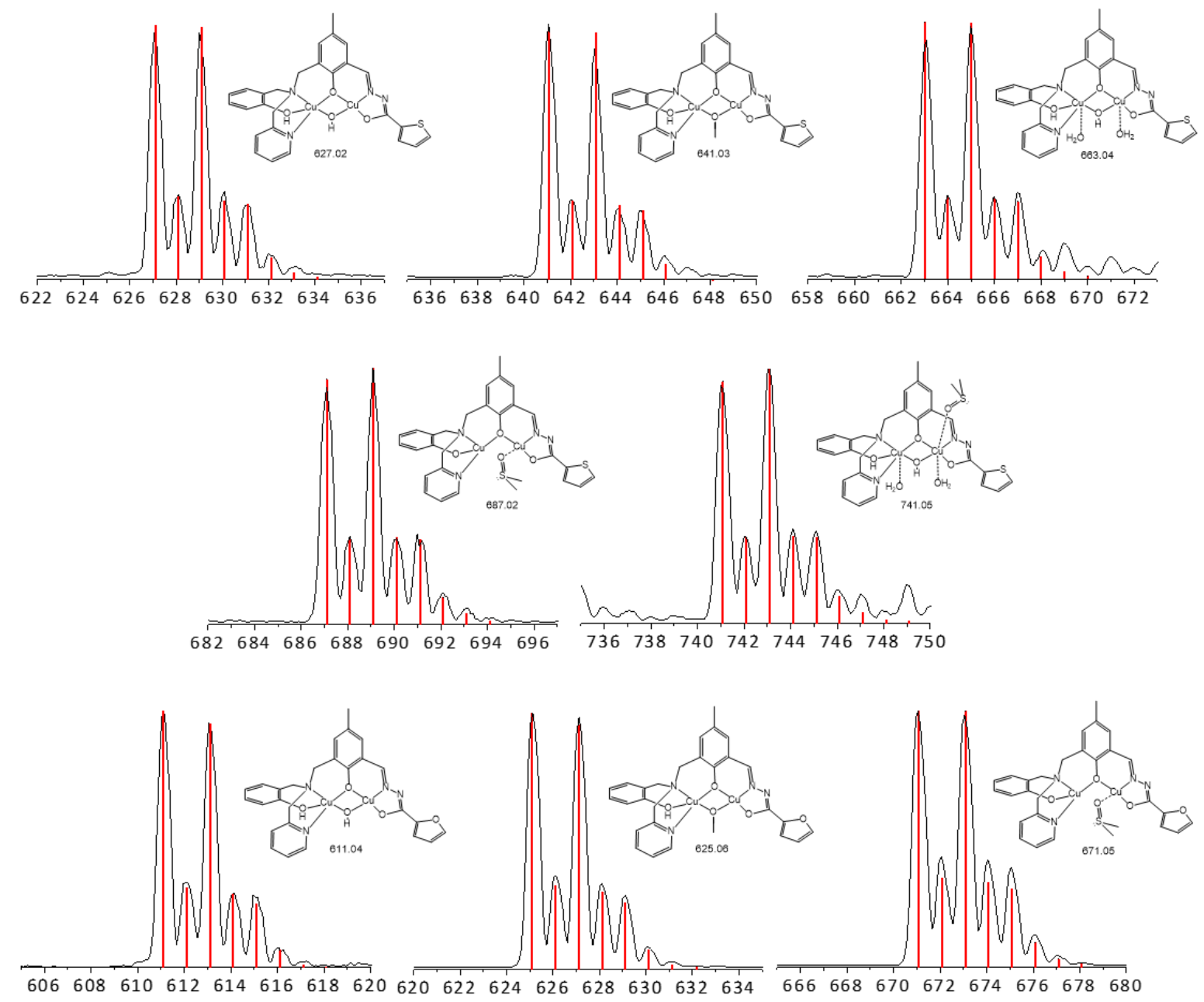

Figure S19. Isotopic pattern obtained experimentally (black curve) and simulated by the Isotope Distribution Calculator and Mass Spec Plotter tool (red vertical lines) for $\mathbf{1}$ and 2. 


\section{Scattering studies.}
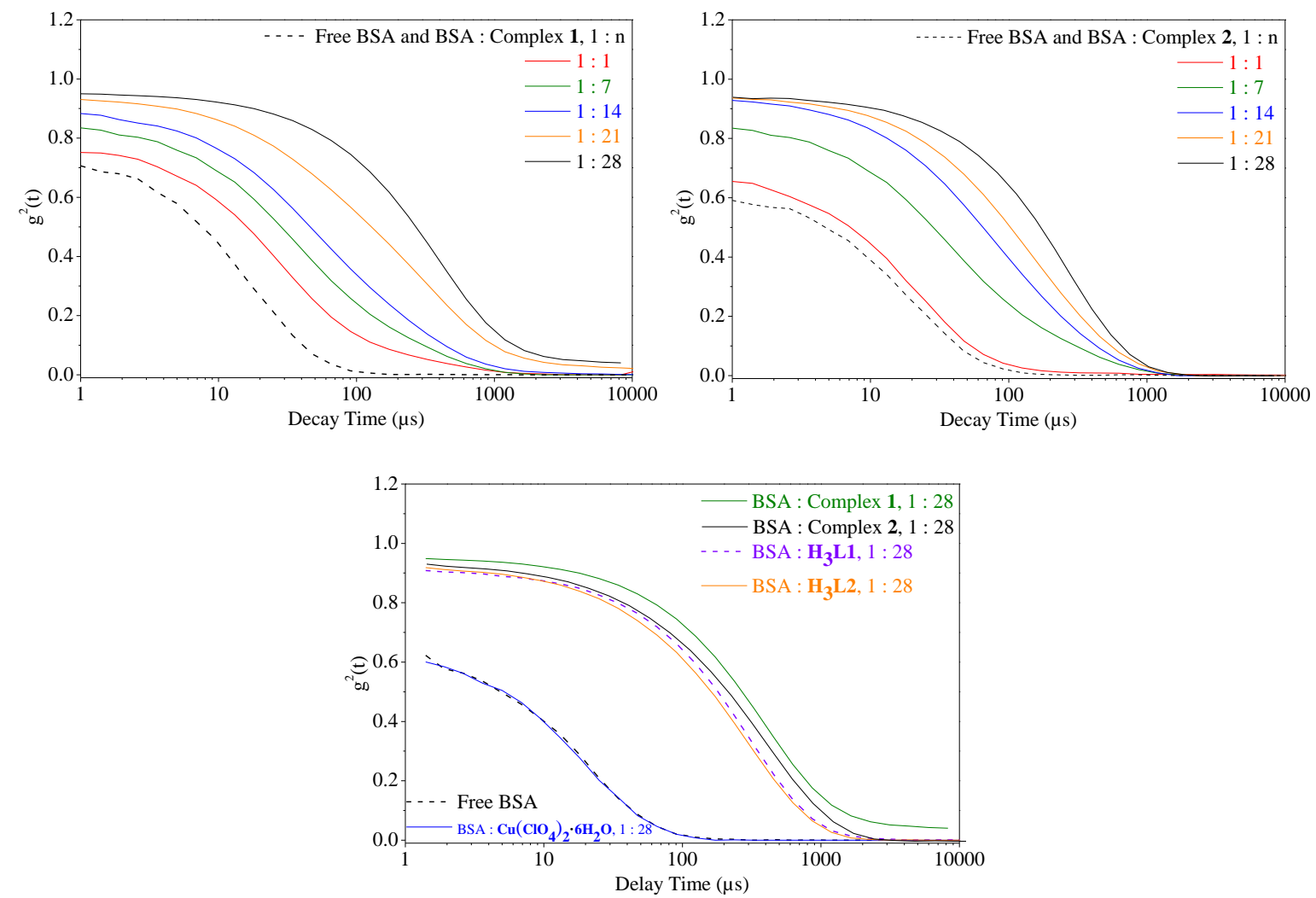

Figure S20. Auto-correlation functions of BSA samples $\left(5 \times 10^{-5} \mathrm{M}\right)$ in the presence of $\mathbf{1}$ and 2 at different molar ratios, ligands and $\mathbf{C u}\left(\mathrm{ClO}_{4}\right)_{2} \cdot 6 \mathrm{H}_{2} \mathrm{O}$. Scattering angle: $90^{\circ} .25^{\circ} \mathrm{C}$.

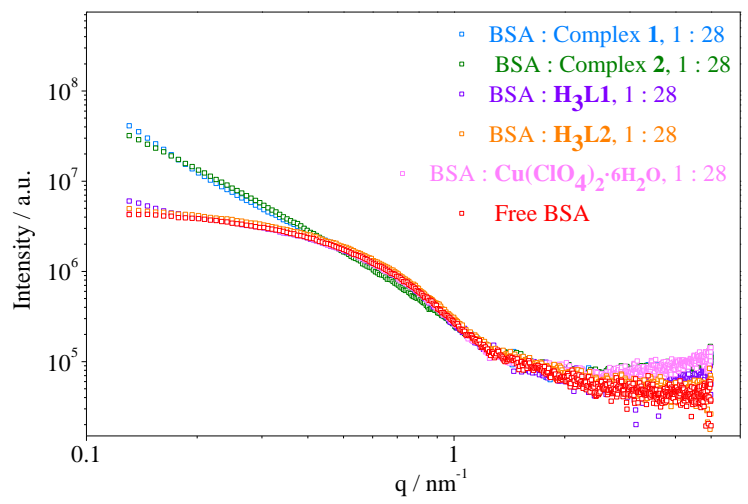

Figure S21. SAXS curves of aqueous solutions containing pure BSA $\left(5 \times 10^{-5} \mathrm{M}\right)$ and BSA in the presence of 28-fold excess 1, 2, $\mathrm{H}_{3} \mathrm{L1}, \mathrm{H}_{3} \mathrm{~L} 2$, and $\mathrm{Cu}\left(\mathrm{ClO}_{4}\right)_{2} \cdot \mathbf{6 H}_{2} \mathrm{O} .25{ }^{\circ} \mathrm{C}$. 

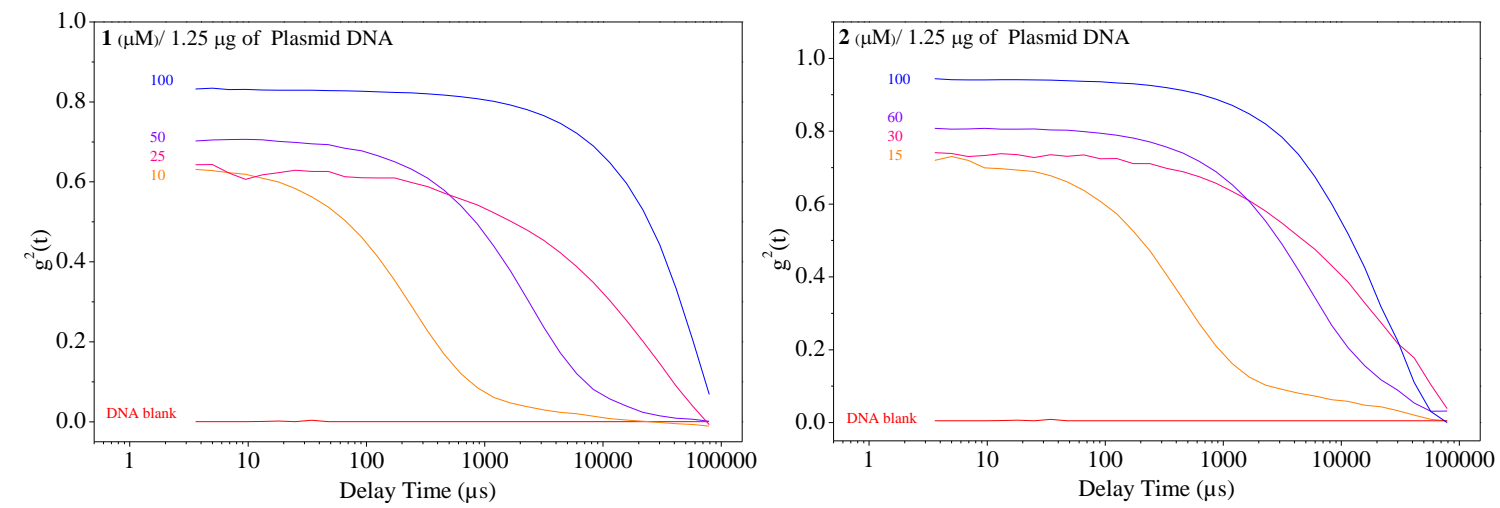

Figure S22. Auto-correlation functions of samples of DNA $(1.25 \mu \mathrm{g}$ in $0.5 \mathrm{~mL})$ in the presence of $\mathbf{1}$ and $\mathbf{2}$ at different molar ratios. Scattering angle: $90^{\circ} .25^{\circ} \mathrm{C}$.

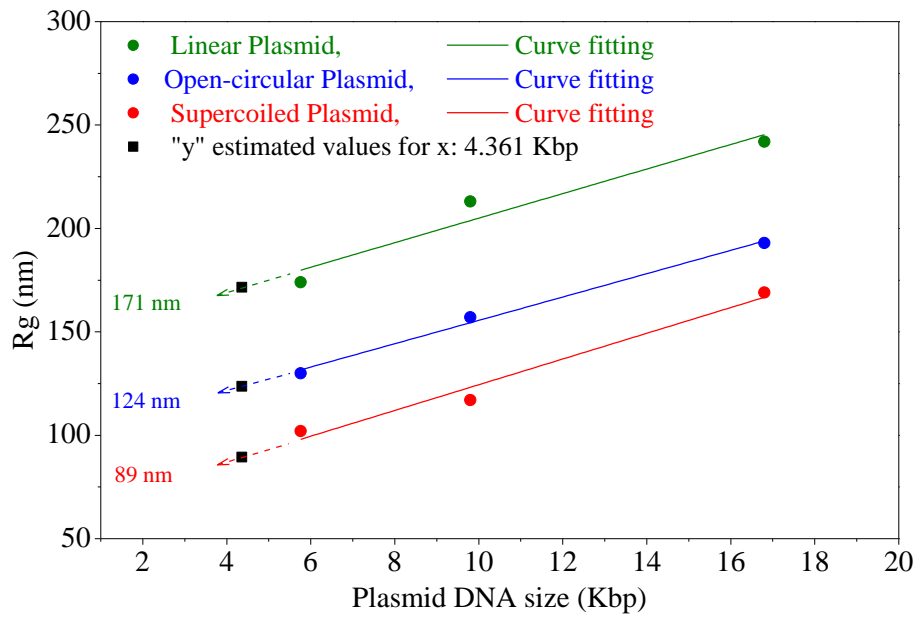

Figure S23. Estimation of the different plasmid pBR322 forms radii by extrapolation, based in the summary reported ${ }^{1}$ by Latulippe and Zydney of $R_{g}$ values from static light scattering analysis of supercoiled, open-circular, and linear isoforms of 5.76, 9.80, and 16.8 kbp plasmids in $10 \mathrm{mM} \mathrm{Na} 2 \mathrm{EDTA}, 200 \mathrm{mM} \mathrm{NaCl}$ solution.

(1) Latulippe, D. R.; Zydney, A. L. Radius of gyration of plasmid DNA isoforms from static light scattering. Biotechnology and Bioengineering. 2010, 107, 134-142. 10.1002/bit.22787 